\title{
SIMULATING THE HOT X-RAY EMITTING GAS IN ELLIPTICAL GALAXIES
}

\author{
Daisuke Kawata and Brad K. Gibson \\ Centre for Astrophysics $\&$ Supercomputing, Swinburne University, Australia \\ dkawata,bgibson@astro.swin.edu.au
}

\begin{abstract}
We study the chemo-dynamical evolution of elliptical galaxies and their hot X-ray emitting gas using high-resolution cosmological simulations. Our Tree N-body/SPH code includes a self-consistent treatment of radiative cooling, star formation, supernovae feedback, and chemical enrichment. We present a series of $\Lambda \mathrm{CDM}$ cosmological simulations which trace the spatial and temporal evolution of heavy element abundance patterns in both the stellar and gas components of galaxies. X-ray spectra of the hot gas are constructed via the use of the vmekal plasma model, and analysed using XSPEC with the XMM EPN response function. Simulation end-products are quantitatively compared with the observational data in both the X-ray and optical regime. We find that radiative cooling is important to interpret the observed $\mathrm{X}$-ray luminosity, temperature, and metallicity of the interstellar medium of elliptical galaxies. However, this cooled gas also leads to excessive star formation at low redshift, and therefore results in underlying galactic stellar populations which are too blue with respect to observations.
\end{abstract}

\section{Introduction}

The hot X-ray emitting gas of elliptical galaxies represents an important interface between galaxies and the intergalactic medium (perhaps even the primary interface). The X-ray halos of ellipticals carry with them two fundamental mysteries:

- their X-ray luminosities are lower than that expected from an extrapolation of the cluster X-ray luminosity-temperature $\left(\mathrm{L}_{\mathrm{X}}-\mathrm{T}_{\mathrm{X}}\right)$ relation (e.g. Matsushita et al. 2000).

- their X-ray metallicities are lower than that of the mean stellar iron abundance (the so-called "iron discrepancy" - e.g. Arimoto et al. 1997 - a "discrepancy" in the sense that the halo gas metallicity was expected to exceed that of the stars, since it should bear 


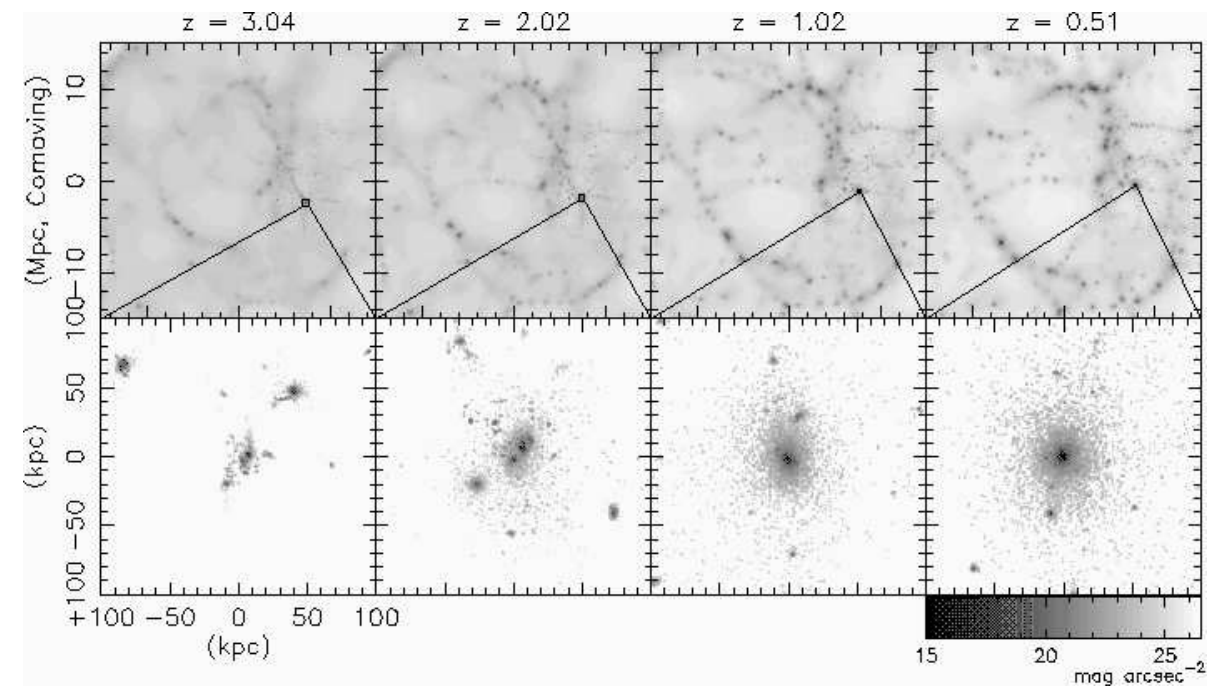

Figure 1 Dark matter density map of a portion of the $43 \mathrm{Mpc}$ (comoving) simulation volume (upper panels), and predicted $I$-band image of the target galaxy (lower panels), over the redshift range $z=3.0$ to $z=0.5$.

the pollution of the enrichment from earlier generation of stars enrichment byproducts that were not locked up into subsequent stellar generations). ${ }^{1}$

Conversely, the optical properties of ellipticals appear less contentious! The Colour-Magnitude Relation (CMR) and Fundamental Plane provide strong constraints for any elliptical galaxy formation paradigm. We present here our preliminary work aimed ultimately at the construction of successful self-consistent optical + X-ray cosmological chemodynamical simulations of elliptical galaxies.

\section{Methods}

In our simulations, the dynamics of collisionless dark matter and stars is calculated using a gravitational Tree N-body code, and the gas component is modeled using Smoothed Particle Hydrodynamics (SPH). We calculate radiative cooling, star formation, chemical enrichment, and supernovae (SNe) feedback, self-consistently, and take into account both

\footnotetext{
${ }^{1}$ X-ray iron abundances remain a controversial issue (c.f. Buote \& Fabian 1997), although the iron discrepancy appears to hold based upon recent high-resolution XMM RGS observations (Xu et al. 2002; Sakelliou et al. 2002).
} 
Type Ia and Type II SNe. We assume that SNe feedback is released as thermal energy. Details of the code are presented in Kawata (2001) and Kawata \& Gibson (2003, in prep).

We have carried out a series of high-resolution simulations within the adopted standard $\Lambda$ CDM cosmology $\left(\Omega_{0}=0.3, \Lambda_{0}=0.7, \Omega_{\mathrm{b}}=0.019 h^{-2}\right.$, $h=0.7$, and $\left.\sigma_{8}=0.9\right)$. Gas dynamics and star formation are included only within the relevant high-resolution region $(\sim 12 \mathrm{Mpc}$ at $z=0)$; the surrounding low-resolution region $(\sim 43 \mathrm{Mpc})$ contributes to the highresolution region only through gravity. The mass of individual gas particles in the high-resolution region was $5.9 \times 10^{7} \mathrm{M}_{\odot}$. We next identified an appropriate elliptical galaxy analog in the high-resolution region, which acts as the focus for this preliminary study. The total virial mass of this target galaxy is $2 \times 10^{13} \mathrm{M}_{\odot}$, similar in size to that of NGC 4472, a bright elliptical galaxy in the Virgo Cluster. The target galaxy is relatively isolated, with only a few low-mass satellites remaining at $z=0$.

Figure 1 shows the morphological evolution of dark matter in the simulation volume, and the evolution of the stellar component in a $200 \mathrm{kpc}$ region centred on the target galaxy. The galaxy forms through conventional hierarchical clustering between redshifts $z=3$ and $z=1$; the morphology has not changed dramatically since $z=1$. Three different radiative cooling and SNe feedback models were considered: Model A is an adiabatic model (i.e. no cooling); Model B includes cooling and weak feedback; Model C mimics Model B, but incorporates stronger feedback (100 times larger thermal energy per supernova).

For all the models, we examine both the resulting X-ray and optical properties, comparing them quantitatively with observation. The gas particles in our simulations carry with them knowledge of the density, temperature, and abundances of various heavy elements in their immediate vicinity. Using the XSPEC vmekal plasma model, we derive the $\mathrm{X}$-ray spectra for each gas particle, and synthesize them within the assumed aperture $(\mathrm{R} \sim 20 \mathrm{kpc})$. We next generate "fake" spectra with the response function of XMM EPN detector, assuming an exposure time (40 ks) and target galaxy distance $(17 \mathrm{Mpc})$. Finally, our XPSEC fitting provides the X-ray weighted temperatures and abundances of various elements. Conversely, the simulated star particles each carry their own age and metallicity "tag", which enables us to generate an optical-to-near infrared spectral energy distribution for the target galaxy, when combined with our population synthesis code adopting simple stellar populations of Kodama \& Arimoto (1997). 

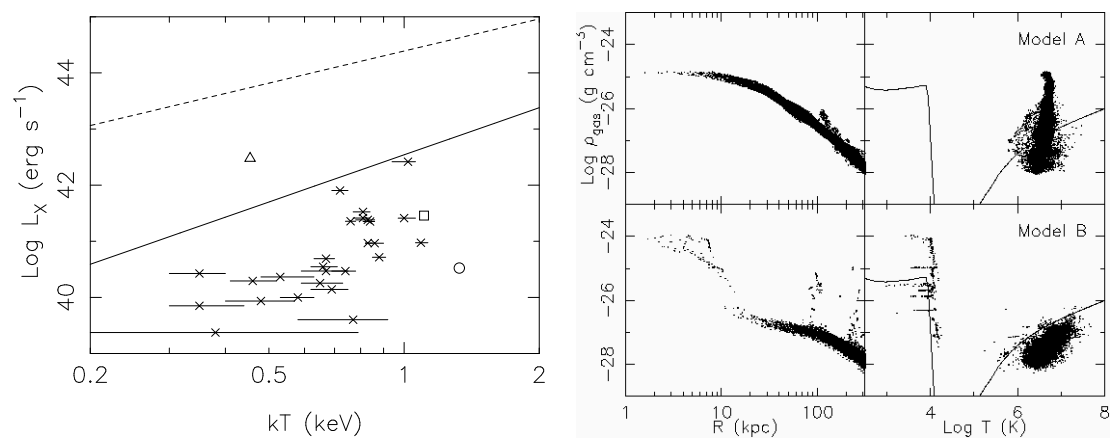

Figure 2 Left panel: Comparison of the simulated and observed (crosses with error bars) $\mathrm{L}_{\mathrm{X}}-\mathrm{T}_{\mathrm{X}}$ relations. The triangle/circle/square indicates the predictions of Model A/B/C. The solid line indicates the extrapolation of the cluster $\mathrm{L}_{\mathrm{X}}-\mathrm{T}_{\mathrm{X}}$ relation (Edge \& Stewart 1991), while the dashed line represents the relation obtained from the adiabatic simulation of Muanwong et al. (2001). Right panel: Density vs radius (left) and density vs temperature (right) distributions of gas particles for Model A (upper) and Model B (lower). The sold curves separate the region where the cooling time is shorter (upper region) and longer than the Hubble time.

\section{Results and Conclusion}

The left panel of Figure 2 shows the predicted $\mathrm{L}_{\mathrm{X}}-\mathrm{T}_{\mathrm{X}}$ relation for the three models at $z=0$; crosses with error bars represent the observational data from Matsushita et al. (2000). The adiabatic model (Model A) appears incompatible with the data due to its excessive luminosity and low temperature. The inclusion of radiative cooling leads to lower luminosities and higher temperatures - as a result, models with cooling (Models B and C) are (roughly) consistent with the $\mathrm{L}_{\mathrm{X}}-\mathrm{T}_{\mathrm{X}}$ relation of the observed elliptical galaxies. These conclusions are consistent with the analysis of Muanwong et al. (2001). The right panel of Figure 2 shows the effect of cooling more clearly. In the gas density versus temperature diagrams, the region above than the line corresponds to a parameter space in which the cooling time is shorter than the Hubble time. Cooling ensures the gas within this region is cold (ie. non X-ray emitting), and of low density and high temperature, ensuring that ultimately radiative cooling drives the observed $\mathrm{L}_{\mathrm{X}}-\mathrm{T}_{\mathrm{X}}$ relation.

The left panel of Figure 3 compares the X-ray weighted iron abundance of our simulations with the observational data of Matsushita et al. (2000). As the adiabatic model (by construction) does not form any stars (not having any cooling!), we show the results only for Models B and 

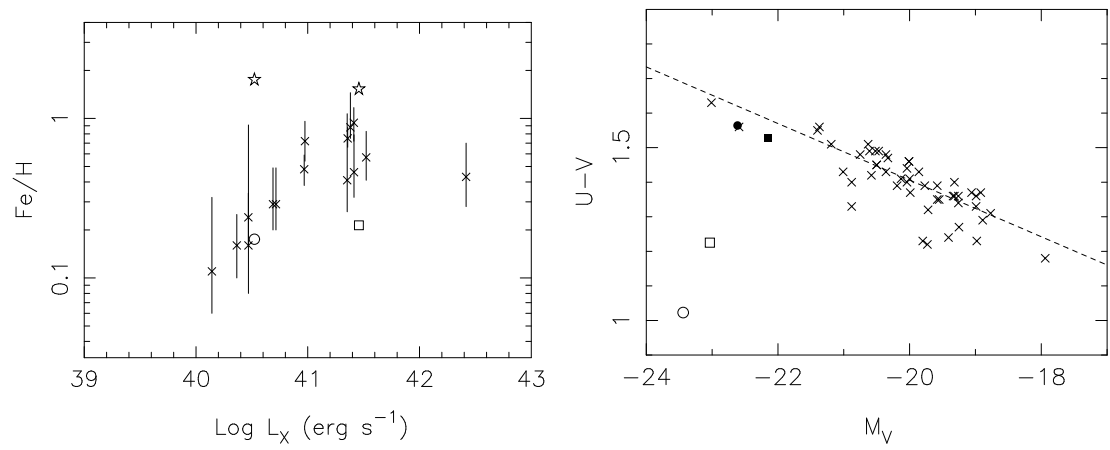

Figure 3 Left panel: Comparison of the simulated and observed (crosses with error bars) $[\mathrm{Fe} / \mathrm{H}]_{\mathrm{X}}-\mathrm{L}_{\mathrm{X}}$ relations. The open circle/square shows the predictions of Model B/C; open stars show the predicted mean metallicity of the stellar component for Models B and C. Right panel: Comparison of the simulated CMRs (open circle/square for Model B/C) and that of the Coma cluster ellipticals (crosses). The dashed line shows the CMR fitted to the Coma Cluster galaxies. The solid circle/square demonstrates the colour and magnitude for Model B/C when the contribution from young stars (age $<8 \mathrm{Gyr}$ ) is ignored.

C. Both these models show lower gas-phase iron abundance, compared to their stellar abundance, consistent with the low iron abundances observed in the X-ray emitting gas of ellipticals. We find that a large fraction of iron ejected from stars is locked into future generation of stars. Stars preferentially enrich the gas in the central region, where cooling is efficient (right panel of Figure 2). The enriched gas can then cool easily and be incorporated into future generations of stars. Consequently, the hot gaseous halo has not been enriched efficiently, leading to a lower X-ray weighted iron abundance.

In summary, our radiative cooling models explain the two X-ray "mysteries" alluded to in Section 1. Having said that, any successful scenario must also explain the optical properties of the underlying stellar component. To this end, we examined the position of our simulated target galaxy in the observed Coma cluster CMR (Bower et al. 1992). We can see immediately that the colours of the resulting stellar components of both Models $\mathrm{B}$ and $\mathrm{C}$ are inconsistent with the data (being too blue). This inconsistency can be traced to an excessive population of young and intermediate age stars ( $<8$ Gyr) which form from successively cooled gas, regardless of the strength of $\mathrm{SNe}$ feedback. If the contribution of these young stars was ignored, the resulting colours would match the observed CMR. Therefore, one exotic (if somewhat ad hoc) solution is to "hide" 
these younger stars within a bottom-heavy initial mass function (IMF) such that they cannot be seen today even if they did exist (e.g. Fabian et al. 1982; Mathews \& Brighenti 1999). Another (more plausible) possibility is that extra heating sources, such as intermittent AGN activity, suppress star formation at low redshift. Before suggesting this is the true solution though, we must re-examine the predicted X-ray properties of the simulation end-products after introducing these additional heating sources; we will be pursuing this comparison in a future paper.

Our cosmological chemodynamical code makes it possible to undertake quantitative comparisons between numerical simulations and observational data in both the X-ray and optical regime with minimal assumptions. We find that radiative cooling is required to explain the observed X-ray luminosity, temperature, and metallicity of elliptical galaxies. Unfortunately, the resulting cooled gas also leads to unavoidable overproduction of young and intermediate age stellar populations, at odds with the observational constraints. Although a bottom-heavy IMF is one solution for this problem, extra heating by intermittent AGN activity seems to be more plausible (e.g. Brighenti \& Mathews 2002); recent observations are consistent with this latter picture (e.g. Churazov et al. 2001).

\section{Acknowledgements}

We acknowledge the support of the Australian Research Council through the Large Research Grant Program (A0010517) and Swinburne University through the Research Development Grants Scheme.

\section{References}

Arimoto, N., Matsushita, K., Ishimaru, Y., et al., 1997, ApJ, 477, 128

Bower, R.G., Lucey, J..R., \& Ellis, R.S., 1992, MNRAS, 254, 589

Brighenti, F., \& Mathews, W.G., 2002, ApJL, 574, L11

Buote, D.A., Fabian, A.C., 1998, MNRAS, 296, 977

Churazov E., Brüggen, M., Kaiser, C.R., et al., 2001, ApJ, 554, 261

Edge, A.C., \& Stewart, G.C., 1991, MNRAS, 252, 414

Fabian, A.C., Nulsen, P.E.J., \& Canizares, C.R., 1982, MNRAS, 201, 933

Kawata, D., 2001, ApJ, 558, 598

Kodama, T., \& Arimoto, N., 1997, A\&A, 320, 41

Matsushita, K., Ohashi, T., \& Makishima, K., 2000, PASJ, 52, 685

Mathews, W.G., \& Brighenti, F., 1999, ApJ, 526, 114

Muanwong, O., Thomas, P.A., Kay, S.T., et al., 2001, ApJL, 552, L27

Sakelliou, I. et al., 2002, A\&A, 391, 903

$\mathrm{Xu}$, H. et al., 2002, ApJ, 579, 600 